\title{
Uso de dispositivos mecânicos de compressão torácica na parada cardíaca: uma revisão de literatura
}

\author{
Use of mechanical chest compression devices in cardiac arrest: a literature review
}

Uso de dispositivos mecánicos de compresión torácica en paro cardíaco: revisión de la literatura

Tales de Sousa Coutinho Ferreira Pires ${ }^{1 *}$, Eloá Costa Cândido Fontana², Carlos Eduardo Rocha Pinto ${ }^{1}$, Gustavo Campos de Farias Kifer Moreira Ribeiroํ, Bruno Cezario Costa Reis ${ }^{1}$.

\section{RESUMO}

Objetivo: Avaliar de forma abrangente se os dispositivos mecânicos de RCP podem trazer melhores resultados clínicos do que a compressão torácica manual. Métodos: Trata-se de uma revisão bibliográfica narrativa. Um protocolo de pesquisa foi formalizado e executado para conduzir a pesquisa no banco de dados do PubMed durante julho de 2020 e setembro de 2020, com artigos publicados entre o ano de 2015 e 2020. Os resultados obtidos foram analisados e incorporados ao presente estudo a partir de critérios de inclusão e exclusão estabelecidos no protocolo. Resultados: Buscou-se elucidar acerca do uso de dispositivos mecânicos na RCP e a técnica convencional, feita por compressão manual e como resultado constatou-se que apesar de um bom desempenho durante a compressão torácica, os dispositivos mecânicos não se mostraram mais efetivos que a técnica manual, pois não dependem apenas de fatores técnicos, mas também de fatores relacionados ao procedimento e a organização da equipe. Considerações finais: Uma boa indicação para o uso desses dispositivos mecânicos, quando utilizados por indivíduos capacitados, são em situações onde não são praticáveis as compressões torácicas manuais consistentes ou quando elas são perigosas para o socorrista.

Palavras-chave: Parada cardíaca, Reanimação cardiopulmonar, Massagem cardíaca.

\begin{abstract}
Objective: To comprehensively assess whether mechanical CPR devices can bring better clinical results than manual chest compression. Methods: This is a narrative bibliographic review. A research protocol was formalized and executed to conduct the search in the PubMed database during July 2020 and September 2020, with articles published between 2015 and 2020. The results obtained were analyzed and incorporated into the present study from inclusion and exclusion criteria established in the protocol. Results: We sought to elucidate about the use of mechanical devices in CPR and the conventional technique, performed by manual compression and as a result it was found that despite a good performance during chest compression, the mechanical devices were not more effective than the manual technique, as they depend not only on technical factors, but also on factors related to the procedure and the organization of the team. Final considerations: A good indication for the use of these mechanical devices, when used by trained individuals, are in situations where consistent manual chest compressions are not practicable or when they are dangerous for the rescuer.
\end{abstract}

Keywords: Cardiac arrest, Cardiopulmonary resuscitation, Heart massage.

\footnotetext{
1 Universidade de Vassouras (UV), Vassouras - RJ. *E-mail: talessc3@hotmail.com

2 Universidade Estácio de Sá (UNESA-PV), Rio de Janeiro - RJ.
}

SUBMETIDO EM: 12/2020

ACEITO EM: 1/2021

PUBLICADO EM: 2/2021 


\section{RESUMEN}

Objetivo: Evaluar exhaustivamente si los dispositivos mecánicos de RCP pueden brindar mejores resultados clínicos que la compresión torácica manual. Métodos: Se trata de una revisión bibliográfica narrativa. Se formalizó y ejecutó un protocolo de investigación para realizar la búsqueda en la base de datos PubMed durante julio de 2020 y septiembre de 2020, con artículos publicados entre 2015 y 2020. Los resultados obtenidos fueron analizados e incorporados al presente estudio de criterios de inclusión y exclusión establecidos en el protocolo. Resultados: Se buscó dilucidar sobre el uso de dispositivos mecánicos en RCP y la técnica convencional, realizada por compresión manual y como resultado se encontró que a pesar de un buen desempeño durante la compresión torácica, los dispositivos mecánicos no fueron más efectivos que los técnica manual, ya que dependen no solo de factores técnicos, sino también de factores relacionados con el procedimiento y la organización del equipo. Consideraciones finales: Una buena indicación para el uso de estos dispositivos mecánicos, cuando los utilizan personas capacitadas, es en situaciones en las que no se pueden realizar compresiones torácicas manuales consistentes o cuando son peligrosas para el rescatista.

Palabras clave: Paro cardíaco, Reanimación cardiopulmonar, Masaje cardíaco.

\section{INTRODUÇÃO}

A parada cardíaca é uma diminuição repentina da atividade cardíaca que prejudica o bombeamento eficaz do sangue. Mais de 420.000 pessoas sofrem uma parada cardíaca extra-hospitalar nos Estados Unidos a cada ano, e a American Heart Association (AHA) estima que esse número aumentará nos próximos anos. Nos últimos 50 anos, as pesquisas têm melhorado continuamente reanimação cardiopulmonar (RCP), mas ainda há muito a fazer, uma vez que as taxas de sobrevida permanecem baixas (LIU M, et al., 2019).

A eficácia da RCP depende de muitos fatores, onde a rapidez e a qualidade do procedimento de ressuscitação são os mais importantes. As diretrizes de ressuscitação cardiopulmonar da AHA de 2015 sugerem que a RCP de alta qualidade tenha as seguintes características: frequência adequada, profundidade suficiente, recuo total do tórax, interrupções mínimas durante as compressões e evitar hiperventilação (KHAN SU, et al., 2018; ZHU N, et al., 2019).

A RCP precoce e de alta qualidade está intimamente relacionada à taxa de sobrevida de pacientes com parada cardíaca. Se a RCP for realizada imediatamente, a taxa de sobrevida dos pacientes é de $63,6 \%$. Se a RCP for realizada em 5 minutos, a sobrevida é de $37,5 \%$. No entanto, se o tempo de RCP for superior a 10 minutos, a taxa de sobrevivência é de apenas 4,5\% (LIU M, et al., 2019).

A vantagem da compressão manual como um método tradicional de compressão torácica é que ela pode ser usada para intervir rapidamente no resgate de um paciente com parada cardíaca extra-hospitalar, sem a necessidade de qualquer assistência mecânica, no entanto, a qualidade da compressão é prejudicada pela fadiga após duração prolongada. Com isso, os socorristas precisam se alternar com frequência, interrompendo a massagem e, consequentemente, reduzindo ainda mais a eficácia da ressuscitação (ZHU N, et al., 2019).

Dispositivos de RCP mecânicos automáticos foram desenvolvidos para resolver alguns problemas que reduzem a eficácia da RCP manual. O primeiro desses problemas, provavelmente o mais importante, é a fadiga que os socorristas sentem durante a RCP (MARTI J, et al., 2017).

Esses dispositivos mecânicos economizam mão de obra, não sofrem fadiga, mantém a estabilização de parâmetros, como frequência, profundidade, ritmo e também possibilitam continuar a massagem durante a desfibrilação ou outras operações complementares (LIU M, et al., 2019).

As desvantagens da compressão mecânica são que ela requer equipamentos que muitas vezes não estão disponíveis e não são usados em tempo hábil. Para a RCP em pacientes com parada cardíaca extrahospitalar, tanto a compressão manual quanto a mecânica apresentam vantagens e desvantagens e não há consenso sobre seus efeitos e resultados (ZHU N, et al., 2019). 
Os atuais sistemas de compressão mecânica são divididos principalmente em dois tipos. O primeiro é a compressão de ponto, representada pelo equipamento LUCAS ou LUCAS-2 (Lund University Cardiac Assist System), o qual fornece principalmente compressão do esterno inferior. O outro tipo é uma compressão de distribuição de carga, que é representada por Autopulse, o qual distribui uniformemente a pressão por todo o tórax para obter a prensagem tridimensional (ZHU N, et al., 2019).

No entanto, como nenhuma evidência foi mostrada de que os dispositivos mecânicos melhoram o resultado da RCP, a AHA não recomenda seu uso rotineiro. Porém, tais dispositivos são uma alternativa viável quando as compressões manuais de alta qualidade são desafiadoras ou perigosas para o provedor (KHAN SU, et al., 2018).

Este estudo tem como objetivo avaliar de forma abrangente se dispositivos de RCP mecânicos podem trazer melhores resultados clínicos do que a compressão torácica manual por meio de uma revisão de literatura.

\section{MÉTODOS}

Para responder às perguntas especificadas, usamos as palavras-chave selecionadas cardiac arrest; cardiopulmonary resuscitation; mechanical chest compression e o seguinte banco de dados: PubMed (US National Library of Medicine/National Institutes of Health). Foram considerados todos os estudos encontrados na área de pesquisa em questão que foram publicados no período de 2015 até 2020.

A revisão da literatura foi realizada de julho de 2020 a setembro de 2020. A busca para materiais que abordem o uso de dispositivos mecânicos na RCP resultou 1.158 artigos. Do total de artigos coletados, após implementados os critérios de inclusão e exclusão, 20 artigos foram selecionados (Figura 1).

Figura 1 - Fluxograma da seleção dos estudos.

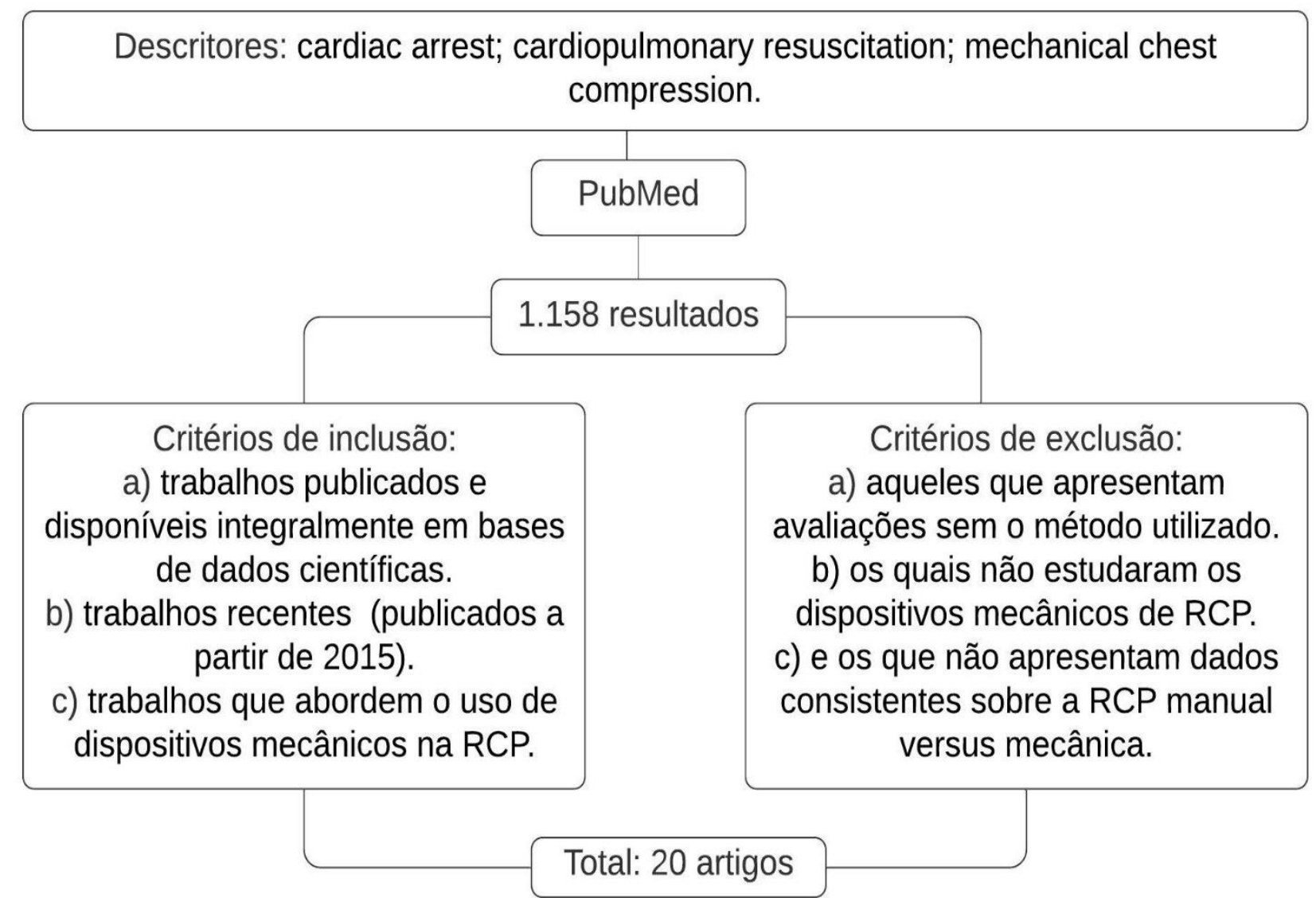

Fonte: Pires TSCF, et al., 2021. 
No protocolo estabelecido para a etapa de planejamento, foi especificado a seguinte questão de pesquisa: "Os dispositivos de RCP mecânicos podem trazer melhores resultados clínicos do que a compressão torácica manual?".

A revisão foi realizada em três etapas: (i) planejamento, em que as diretrizes de pesquisa foram baseadas em um protocolo; (ii) condução, que consistia em executar a busca e seleção de obras de interesse de acordo com a inclusão e exclusão e critérios definidos no protocolo; e finalmente, (iii) a etapa de extração de dados, que permitiu examinar minuciosamente os estudos selecionados, a fim de entender se os dispositivos de RCP mecânicos apresentam melhores resultados clínicos que a compressão torácica manual.

Os critérios utilizados para inclusão de um artigo nesta revisão foram: (a) trabalhos publicados e disponíveis integralmente em bases de dados científicas ou em versões impressas, (b) trabalhos recentes (publicados a partir de 2015) que já possuam aprovação pela comunidade científica, (c) trabalhos que abordem o uso de dispositivos mecânicos na RCP ou que realizaram uma comparação clínica entre a RCP manual versus mecânica, mesmo que este não seja o tema principal do trabalho.

Foram excluídos aqueles que apresentassem avaliações sem o método utilizado, que não estudaram os dispositivos mecânicos de RCP, que não apresentam dados consistentes sobre a RCP manual versus mecânica (Figura 1).

\section{RESULTADOS E DISCUSSÃO}

O Quadro 1 tem por propósito mostrar uma síntese dos artigos analisados, com base em uma revisão bibliográfica, a qual abrange diversos tipos de estudos e análises comparando a RCP por compressão torácica mecânica com a compressão torácica manual.

A análise incluiu os resultados apresentados quanto ao tema abordado. Cada um dos artigos foi analisado individualmente e, posteriormente, foi apresentada uma análise global de todos os trabalhos, levando em consideração os tópicos relevantes sobre o uso de dispositivos mecânicos na RCP. 
Quadro 1 - Resumo dos artigos analisados.

\begin{tabular}{|c|c|c|}
\hline Autor & Objetivo do estudo & Principais resultados \\
\hline $\begin{array}{l}\text { Anatharama } \\
\mathrm{n} \mathrm{V} \text {, et al. } \\
(2017)\end{array}$ & $\begin{array}{l}\text { Determinar se o uso precoce de RCP mecânica, usando um } \\
\text { dispositivo LUCAS 2, resulta em melhores resultados. }\end{array}$ & $\begin{array}{l}\text { Os resultados para RCP manual e RCP com LUCAS precoce e tardio foram, } \\
\text { respectivamente: retorno para circulação espontânea } 28,0 \%, 36,9 \% \text { e } 24,5 \% \text {; } \\
\text { Sobrevivência em } 24 \text { horas } 10,6 \%, 15,5 \% \text { e } 8,2 \% \text {; sobrevivência para alta } 2,9 \% \text {, } \\
5,8 \% \text { e } 2,0 \% \text {; e sobrevida em } 30 \text { dias } 2,4 \%, 5,8 \% \text { e } 0,0 \% \text {. }\end{array}$ \\
\hline $\begin{array}{l}\text { Bonnes JL, et } \\
\text { al. (2016) }\end{array}$ & $\begin{array}{l}\text { Avaliar o efeito da RCP mecânica versus manual nos } \\
\text { resultados clínicos após uma parada cardíaca extra- } \\
\text { hospitalar. }\end{array}$ & $\begin{array}{l}\text { Para a sobrevivência até a admissão hospitalar, os ensaios clínicos randomizados } \\
\text { não indicaram uma diferença entre a RCP mecânica e manual. } \\
\text { Os estudos não randomizados demonstraram um benefício a favor da RCP } \\
\text { mecânica. } \\
\text { A sobrevivência à alta e o resultado neurológico não diferiram entre as estratégias. }\end{array}$ \\
\hline $\begin{array}{l}\text { Couper K, et } \\
\text { al. (2015) }\end{array}$ & $\begin{array}{l}\text { Fornecer novas informações importantes sobre o papel dos } \\
\text { dispositivos mecânicos de compressão torácica. }\end{array}$ & $\begin{array}{l}\text { O uso rotineiro de dispositivos de compressão mecânica no ambiente pré- } \\
\text { hospitalar não melhorou as taxas de sobrevivência. } \\
\text { Estudos observacionais relatam resultados favoráveis com o uso de dispositivos } \\
\text { mecânicos como ponte para terapias avançadas. }\end{array}$ \\
\hline $\begin{array}{l}\text { Couper K, et } \\
\text { al. (2016) }\end{array}$ & $\begin{array}{l}\text { Resumir as evidências em relação ao uso rotineiro de } \\
\text { dispositivos de compressão torácica mecânica durante a } \\
\text { reanimação de uma parada cardíaca hospitalar. }\end{array}$ & $\begin{array}{l}\text { Mostraram uma associação entre o uso de dispositivo de compressão torácica } \\
\text { mecânica e melhora hospitalar ou sobrevida em } 30 \text { dias e sobrevida a curto prazo. } \\
\text { A qualidade geral da evidência em relação a todos os resultados foi muito baixa. }\end{array}$ \\
\hline $\begin{array}{l}\text { Estock JL, et } \\
\text { al. (2016) }\end{array}$ & $\begin{array}{l}\text { Comparar os tempos de interrupção da compressão torácica } \\
\text { necessários para aplicar, ajustar e remover } 2 \text { dispositivos } \\
\text { diferentes de compressão torácica automática. }\end{array}$ & $\begin{array}{l}\text { A interrupção da compressão torácica para aplicar qualquer um dos dispositivos de } \\
\text { compressão torácica ao paciente foi notavelmente maior do que o tempo máximo } \\
\text { de interrupção recomendado pela AHA. }\end{array}$ \\
\hline $\begin{array}{l}\text { Gates S, et } \\
\text { al. (2015) }\end{array}$ & $\begin{array}{l}\text { Resumir as evidências de ensaios clínicos randomizados de } \\
\text { dispositivos mecânicos de compressão torácica usados } \\
\text { durante a ressuscitação após uma parada cardíaca extra- } \\
\text { hospitalar. }\end{array}$ & $\begin{array}{l}\text { Os resultados não mostraram vantagem para o uso de dispositivos mecânicos de } \\
\text { compressão torácica para sobrevivência à alta/30 dias após e sobrevivência com } \\
\text { bom resultado neurológico. }\end{array}$ \\
\hline
\end{tabular}




\begin{tabular}{|c|c|c|}
\hline Autor & Objetivo do estudo & Principais resultados \\
\hline $\begin{array}{l}\text { Gates S, et } \\
\text { al. (2017) }\end{array}$ & $\begin{array}{l}\text { Avaliar o uso do dispositivo LUCAS- } 2 \text { como tratamento de } \\
\text { rotina em serviço de ambulância para parada cardíaca extra- } \\
\text { hospitalar. }\end{array}$ & $\begin{array}{l}\text { A revisão sistemática não encontrou nenhuma evidência de vantagem de } \\
\text { sobrevivência se a compressão torácica mecânica fosse usada. } \\
\text { A análise econômica de saúde mostrou que o LUCAS-2 foi superior a compressão } \\
\text { torácica manual. }\end{array}$ \\
\hline $\begin{array}{l}\text { Khan SU, et } \\
\text { al. (2018) }\end{array}$ & $\begin{array}{l}\text { Comparar a eficácia e segurança relativa dos dispositivos de } \\
\text { compressão mecânica (AutoPulse e LUCAS) com a } \\
\text { compressão manual em pacientes com parada cardíaca. }\end{array}$ & $\begin{array}{l}\text { A compressão manual é mais eficaz do que o AutoPulse e comparável ao LUCAS } \\
\text { na melhora da sobrevida em } 30 \text { dias ou à alta hospitalar e na recuperação } \\
\text { neurológica. } \\
\text { A compressão manual apresentou menor risco de formação de pneumotórax ou } \\
\text { hematoma em comparação com o AutoPulse. }\end{array}$ \\
\hline $\begin{array}{l}\text { Koster RW, } \\
\text { et al. (2017) }\end{array}$ & $\begin{array}{l}\text { Estudar a segurança da compressão torácica mecânica e } \\
\text { determinar possíveis danos em excesso em comparação com } \\
\text { a compressão torácica manual. }\end{array}$ & $\begin{array}{l}\text { LUCAS não causa danos viscerais significativamente mais graves ou com risco de } \\
\text { vida do que a compressão torácica manual. } \\
\text { Para o AutoPulse, o dano visceral não pode ser excluído comparando-se com a } \\
\text { compressão cardíaca manual. }\end{array}$ \\
\hline $\begin{array}{l}\text { Kupershmidt } \\
\text { S, et al. } \\
(2020)\end{array}$ & $\begin{array}{l}\text { Avaliar o impacto do dispositivo LUCAS-2 na sobrevivência no } \\
\text { departamento de emergência em comparação com a RCP } \\
\text { manual, usando um grande conjunto de dados coletados em } \\
\text { um estado principalmente rural dos EUA. }\end{array}$ & $\begin{array}{l}\text { A RCP por compressão manual supera a RCP assistida por automação durante a } \\
\text { parada cardíaca extra-hospitalar. } \\
\text { Os dados também sugerem que o treinamento aprimorado para melhorar os } \\
\text { tempos de resposta e os níveis de experiência com o equipamento pode melhorar } \\
\text { os resultados associados ao LUCAS- } 2 \text {. }\end{array}$ \\
\hline $\begin{array}{l}\text { Liu M, et al. } \\
(2019)\end{array}$ & $\begin{array}{l}\text { Investigar a diferença nos resultados clínicos entre a } \\
\text { compressão torácica manual e a RCP assistida pelo LUCAS } \\
\text { em pacientes com parada cardíaca extra-hospitalar. }\end{array}$ & $\begin{array}{l}\text { Resultados clínicos da RCP com LUCAS e da compressão torácica manual, } \\
\text { respectivamente: retorno a circulação espontânea ( } 33,3 \% \text { vs } 33,0 \%) \text {, sobrevida até } \\
\text { a admissão hospitalar ( } 22,7 \% \text { vs } 24,3 \%) \text {, sobrevida até a alta hospitalar }(8,6 \% \text { vs } \\
10,7 \%) \text { e sobrevida em } 30 \text { dias }(7,5 \% \text { vs } 8,5 \%) \text { e nenhuma diferença significativa } \\
\text { foi encontrada. }\end{array}$ \\
\hline $\begin{array}{l}\text { Marti J, et al. } \\
(2017)\end{array}$ & $\begin{array}{l}\text { Avaliar o custo-benefício do LUCAS- } 2 \text { em comparação com } \\
\text { as compressões torácicas manuais em adultos com parada } \\
\text { cardíaca extra-hospitalar não traumática. }\end{array}$ & $\begin{array}{l}\text { O uso do LUCAS-2 representa um baixo custo-benefício quando comparado à } \\
\text { compressão torácica manual padrão em parada cardíaca extra-hospitalar. }\end{array}$ \\
\hline
\end{tabular}




\begin{tabular}{|c|c|c|}
\hline Autor & Objetivo do estudo & Principais resultados \\
\hline $\begin{array}{l}\text { Milling L, et } \\
\text { al. (2019) }\end{array}$ & $\begin{array}{l}\text { Investigar a extensão e o padrão das lesões relacionadas à } \\
\text { compressão torácica em pacientes submetidos à compressão } \\
\text { cardíaca mecânica e/ou manual. }\end{array}$ & $\begin{array}{l}\text { Lesões viscerais foram mais frequentes em pacientes que receberam compressão } \\
\text { torácica mecânica, mesmo quando ajustados para a duração da compressão, } \\
\text { idade, sexo, índice de massa corporal e terapia anticoagulante. } \\
\text { A ocorrência de lesões em geral foi associada à duração da compressão torácica. }\end{array}$ \\
\hline $\begin{array}{l}\text { Poole K, et } \\
\text { al. (2018) }\end{array}$ & $\begin{array}{l}\text { Fornecer uma atualização sobre uso de dispositivos } \\
\text { mecânicos de RCP na parada cardíaca extra-hospitalar e } \\
\text { hospitalar e fornecer uma visão geral sobre o uso desses } \\
\text { dispositivos em circunstâncias especiais. }\end{array}$ & $\begin{array}{l}\text { Recomenda-se que o uso de dispositivos mecânicos ocorra apenas em sistemas } \\
\text { onde mecanismos de garantia de qualidade estejam em vigor. } \\
\text { Em resumo, os dispositivos mecânicos de RCP podem fornecer um complemento } \\
\text { útil para o tratamento padrão em situações específicas, mas evidências atuais não } \\
\text { suporta seu uso rotineiro. }\end{array}$ \\
\hline $\begin{array}{l}\text { Russo A, et } \\
\text { al. (2017) }\end{array}$ & $\begin{array}{l}\text { Analisar dados recentes sobre a utilização de dispositivos } \\
\text { mecânicos de compressão torácica e esclarecer vantagens e } \\
\text { limitações. }\end{array}$ & $\begin{array}{l}\text { A utilização rotineira de dispositivos mecânicos de compressão torácica não pode } \\
\text { ser recomendada porque estudos randomizados controlados, não demonstraram } \\
\text { sua superioridade em comparação com as compressões torácicas manuais. }\end{array}$ \\
\hline $\begin{array}{l}\text { Tranberg T, } \\
\text { et al. (2015) }\end{array}$ & $\begin{array}{l}\text { Avaliar as compressões torácicas mecânicas fornecidas pelo } \\
\text { LUCAS-2 em comparação com a compressão torácica manual } \\
\text { em uma coorte de casos de parada cardíaca extra-hospitalar. }\end{array}$ & $\begin{array}{l}\text { A fração sem fluxo foi significativamente menor durante a RCP com o LUCAS } \\
(16 \%) \text { do que durante a RCP manual }(35 \%) \text {. }\end{array}$ \\
\hline $\begin{array}{l}\text { Wang PL, et } \\
\text { al. (2018) }\end{array}$ & $\begin{array}{l}\text { Avaliar a eficácia das estratégias de reanimação usando } \\
\text { compressões torácicas mecânicas versus compressões } \\
\text { torácicas manuais padrão com relação à sobrevivência } \\
\text { neurológica em pacientes que sofrem parada cardíaca. }\end{array}$ & $\begin{array}{l}\text { As evidências não sugeriram que os protocolos de RCP envolvendo dispositivos } \\
\text { mecânicos de compressão torácica sejam superiores à terapia convencional } \\
\text { envolvendo apenas compressões torácicas manuais. }\end{array}$ \\
\hline $\begin{array}{l}\text { Wroe PC, et } \\
\text { al. (2018) }\end{array}$ & $\begin{array}{l}\text { Estudar o uso de RCP mecânica em nosso departamento de } \\
\text { emergência }\end{array}$ & $\begin{array}{l}\text { Em } 38 \% \text { dos casos, pelo menos um caso apresentou de falha do dispositivo. } \\
\text { Além disso, } 50 \% \text { apresentaram uma falha recorrente do dispositivo } \\
\text { A duração média das pausas de RCP envolvendo falha do dispositivo foi de } 24 \mathrm{~s} \text {, } \\
\text { em comparação com } 14 \mathrm{~s} \text { para pausas sem falha do dispositivo. }\end{array}$ \\
\hline $\begin{array}{l}\text { Zhu N, et al. } \\
(2019)\end{array}$ & $\begin{array}{l}\text { Avaliar os efeitos da reanimação da compressão torácica } \\
\text { mecânica e manual em pacientes com parada cardíaca extra- } \\
\text { hospitalar. }\end{array}$ & $\begin{array}{l}\text { Não houve diferenças significativas nos efeitos de ressuscitação da compressão } \\
\text { torácica mecânica e manual em termos da taxa de retorno a circulação espontânea, } \\
\text { de sobrevivência à admissão hospitalar e sobrevivência à alta hospitalar, e função } \\
\text { neurológica. }\end{array}$ \\
\hline
\end{tabular}

Fonte: Pires TSCF, et al., 2020. 
Em seu estudo, Gates S, et al. (2015) analisou cinco ensaios, dos quais três avaliaram o dispositivo LUCAS ou LUCAS-2 e dois avaliaram o dispositivo AutoPulse. Os resultados não mostraram vantagem para o uso de dispositivos mecânicos de compressão torácica para sobrevivência à alta hospitalar/30 dias após e sobrevivência com bom resultado neurológico. Em outra pesquisa, Gates S, et al. (2017) concluiu que não houve evidência de melhora na sobrevida em 30 dias com LUCAS-2 em comparação com compressões manuais. Na revisão sistemática de estudos randomizados, não foi sugerido que a sobrevivência ou sobrevivência sem deficiência significativa possa ser melhorada com o uso de compressão torácica mecânica.

A análise realizada por Marti J, et al. (2017) incluiu 4.471 pacientes no estudo (1.652 designados para o grupo LUCAS-2, 2.819 designados para o grupo de controle). Aos 12 meses, 89 (5\%) pacientes sobreviveram no grupo LUCAS-2 e 175 (6\%) sobreviveram no grupo de RCP manual. Portanto, os pacientes no grupo LUCAS-2 tiveram resultados de saúde piores e incorreram em custos de saúde e assistência social mais elevados. Com isso, o estudo demonstra que o uso do dispositivo de compressão torácica mecânica LUCAS2 representa um baixo custo-benefício quando comparado à compressão torácica manual padrão em parada cardíaca extra-hospitalar. Os resultados são impulsionados por piores desfechos neurológicos e menor sobrevida no grupo LUCAS-2 em comparação com a RCP manual

De acordo com Khan SU, et al. (2018) foram analisados 12.908 pacientes com parada cardíaca, sendo 2.608 pacientes reanimados com AutoPulse, 3.308 com LUCAS e 6.992 com compressão manual. A compressão manual melhorou a sobrevida à alta hospitalar, em 30 dias e a recuperação neurológica em comparação com AutoPulse. Porém, não houve diferenças entre o LUCAS e o AutoPulse no que diz respeito à sobrevivência à admissão hospitalar, recuperação neurológica ou retorno da circulação espontânea. Além disso, a compressão manual reduziu o risco de pneumotórax, enquanto a compressão manual e o LUCAS reduziram o risco de formação de hematoma em comparação com o AutoPulse. A análise de probabilidade classificou a compressão manual como o tratamento mais eficaz para melhorar a sobrevida em 30 dias ou à alta hospitalar e apresentou menor risco de formação de pneumotórax e hematoma.

O estudo realizado por Khan SU, et al. (2018) ainda afirma que a AHA considera a compressão mecânica aceitável para continuar a RCP durante o transporte ou durante a revascularização coronária, no entanto, os dispositivos não apresentam benefício de sobrevivência. As possíveis explicações para esta observação são múltiplas, como o uso do dispositivo podendo prolongar o tempo até a primeira aplicação do choque. O estudo pioneiro ASPIRE (Auto Pulse Assisted Prehospital International Resuscitation), o tempo médio para o primeiro choque na fibrilação ventricular foi prolongado, ocorrendo em 2,1 minutos.

Ademais Khan SU, et al. (2018) referiu também outros fatores relacionados com o uso dos dispositivos mecânicos, que não indicam seu uso rotineiro, sendo um deles que nem todos os pacientes podem ser adaptados aos dispositivos mecânicos disponíveis. Em um estudo, 3,5\% dos pacientes não conseguiram ser ajustado ao dispositivo e apenas $95 \%$ dos pacientes puderam receber o dispositivo LUCAS. Devido a esse fator, pode atrasar ainda mais a implantação do dispositivo e, portanto, comprometer os resultados. Por último, o feedback da qualidade da RCP não foi adequado em alguns dos estudos, pois esses dispositivos de feedback são um componente crítico para avaliar a qualidade da RCP e ajudam no ajuste das compressões torácicas.

Wroe PC, et al. (2018) revisou 18 meses de gravações de vídeo de ressuscitações de parada cardíaca em um departamento de emergência onde usavam um sistema de compressão torácica mecânica (LUCAS 2). Observou-se que em $80 \%$ dos casos foi usada a RCP mecânica e 38\% destes envolveram pelo menos um caso de falha do dispositivo e $50 \%$ envolveram uma falha recorrente do dispositivo. Além disso, a duração média das pausas de RCP envolvendo falha do dispositivo foi de $24 \mathrm{~s}$, em comparação com $14 \mathrm{~s}$ para pausas sem falha do dispositivo. Ainda relatou que as falhas mais comuns foram problemas com a colocação, posicionamento ou reposicionamento do dispositivo e dificuldade para iniciar o dispositivo e também foi observado vários casos de desprendimento espontâneo.

Outrossim, Wroe PC, et al. (2018) referiu que o uso mais criterioso do dispositivo pode diminuir a frequência de falhas no departamento de emergência e que os provedores devem estar cientes das armadilhas potenciais para a implantação do dispositivo e deve considerar seus riscos e benefícios antes de 
implementá-lo. Contudo, considerando a alta taxa de falha recorrente do dispositivo, os provedores devem considerar abandoná-lo no início de uma ressuscitação caso ocorra uma falha inicial.

Liu M, et al. (2019) em seu estudo envolveu 8.501 indivíduos para analisar os resultados clínicos da RCP com LUCAS e da compressão torácica manual, respectivamente, para pacientes com parada cardíaca extrahospitalar. Comparações como retorno a circulação espontânea (33,3\% vs 33,0\%), sobrevida até a admissão hospitalar (22,7\% vs $24,3 \%)$, sobrevida até a alta hospitalar ( $8,6 \%$ vs $10,7 \%)$ e sobrevida em 30 dias $(7,5 \%$ vs $8,5 \%$ ) foram feitos e nenhuma diferença significativa foi encontrada. A síntese das evidências disponíveis também não suporta que a compressão torácica mecânica com o dispositivo LUCAS melhore o resultado clínico em pacientes com parada cardíaca extra-hospitalar em comparação com a compressão torácica manual.

A comparação feita por Estock JL, et al. (2016) sobre o tempo de interrupção da compressão torácica necessário para aplicar, ajustar e remover 2 dispositivos diferentes de compressão torácica mecânica, mostrou que a interrupção da compressão torácica para aplicar qualquer um dos dispositivos foi notavelmente maior do que o tempo máximo de interrupção recomendado pela AHA.

Segundo Milling L, et al. (2019) a compressão torácica mecânica como adjuvante da compressão torácica manual foi fortemente associada a lesões viscerais potencialmente fatais, sendo a duração da compressão torácica associada à lesão. Os resultados sugerem que a compressão torácica mecânica só deve ser aplicada em situações em que a compressão torácica manual é inviável.

Couper K, et al. (2015) relatou que a RCP com dispositivos mecânicos resulta em taxas de sobrevida semelhantes à RCP manual em parada cardíaca extra-hospitalar e não há dados suficientes para apoiar ou refutar o seu uso de rotina. Contudo, estudos observacionais demonstram a viabilidade do uso de RCP com dispositivos mecânicos quando a RCP manual é difícil ou impossível, e como uma ponte para terapias avançadas.

De acordo com Koster RW, et al. (2017), um total de três pacientes morreram devido a riscos relacionados à ressuscitação: dois pacientes em que foram usados o dispositivo LUCAS tiveram ruptura do fígado e hemorragia maciça e um paciente em que foi usado o dispositivo AutoPulse teve pneumotórax hipertensivo com embolia aérea, que causou acidente vascular cerebral. Além disso, uma taxa mais elevada de lesões viscerais graves ocorreu com AutoPulse $(11,6 \%)$, seguido por LUCAS $(7,4 \%)$ ou compressão manual $(6,4 \%)$. Esses achados levantam questões de segurança, uma vez que essas complicações podem comprometer ainda mais um estado hemodinâmico já gravemente comprometido e pode contribuir para 0 aumento da mortalidade.

Ainda de acordo com a pesquisa de Koster RW, et al. (2017), conclui-se que o dispositivo mecânico de RCP LUCAS não causa danos viscerais significativamente mais graves ou com risco de vida do que a compressão torácica manual. Porém, o dispositivo mecânico AutoPulse, o dano visceral significativamente mais sério ou com risco de vida não pode ser excluído comparando-se com a compressão torácica manual.

Poole K, et al. (2018) afirmou que em situações nas quais as compressões torácicas manuais de alta qualidade não podem ser aplicadas com segurança, o uso de um dispositivo mecânico pode ser uma abordagem clínica razoável, como no transporte de ambulância e na intervenção coronária percutânea primária. Contudo, o processo de implantação requer interrupções na compressão torácica, o que pode ser prejudicial se a pausa for prolongada, por isso recomenda-se que o uso de dispositivos mecânicos ocorra apenas em sistemas onde mecanismos de garantia de qualidade estejam em vigor para monitorar e gerenciar as pausas associadas à implantação. Em resumo, os dispositivos mecânicos de RCP podem fornecer um complemento útil para o tratamento padrão em situações específicas, mas evidências atuais não suportam seu uso rotineiro.

Vinte estudos foram analisados por Bonnes JL, et al. (2016), sendo 5 ensaios clínicos randomizados e 15 estudos não randomizados. Para a sobrevivência até a admissão hospitalar, a estimativa combinada dos ensaios clínicos randomizados não indicou uma diferença entre a RCP mecânica e manual, em contraste, a meta-análise de estudos não randomizados demonstrou um benefício a favor da RCP mecânica e para a 
sobrevivência à alta e o resultado neurológico não diferiram entre as estratégias. Embora existam dados observacionais de qualidade inferior que sugiram que a RCP mecânica possa melhorar a sobrevida até a admissão hospitalar, a evidência cumulativa de alta qualidade randomizada não apoia o seu uso rotineiro para melhorar a sobrevida ou o resultado neurológico. O estudo prospectivo, randomizado e multicêntrico de Anantharaman $\mathrm{V}$, et al. (2017) recrutou 1.274 pacientes, desses 1.191 foram elegíveis para análise, os quais 889 receberam RCP manual e 302 receberam RCP com o LUCAS. Os resultados para RCP manual e RCP com o LUCAS foram: retorno para circulação espontânea 29,2\% e 31,1\%; Sobrevida em 24 horas 11,2\% e 13,2\%; sobrevivência para alta hospitalar 3,6\% e 4,3\%; e sobrevida em 30 dias 3,0\% e 4,0\%, respectivamente.

A pesquisa supracitada feita por Anantharaman $V$, et al. (2017) ainda demonstrou os resultados para RCP manual e RCP com LUCAS precoce e tardio, respectivamente: retorno para circulação espontânea $28,0 \%$, $36,9 \%$ e 24,5\%; Sobrevivência em 24 horas 10,6\%, 15,5\% e 8,2\%; sobrevivência para alta 2,9\%, 5,8\% e $2,0 \%$; e sobrevida em 30 dias 2,4\%, 5,8\% e 0,0\%. Com isso, esse estudo mostrou um benefício de sobrevivência da RCP com o LUCAS em comparação com a manual apenas quando o dispositivo foi aplicado precocemente no local. Zhu N, et al. (2019) também concluiu que não houve diferenças significativas nos efeitos de ressuscitação entre a compressão torácica mecânica e manual em pacientes com parada cardíaca extra-hospitalar. Contudo, para garantir a qualidade da RCP, sugeriu que a compressão torácica manual seja aplicada no estágio inicial da RCP, enquanto a compressão mecânica pode ser usada como parte do suporte avançado de vida no estágio tardio.

Porém, o estudo prospectivo de Tranberg T, et al. (2015), analisou dados de 696 pacientes de parada cardíaca extra-hospitalar não traumática. Destes, 155 foram tratados com o RCP mecânica (LUCAS) após RCP manual. A duração média total do evento foi de 21 minutos, e o tempo com o LUCAS foi significativamente mais longo do que o episódio de RCP manual, 13 minutos versus 5 minutos. A fração sem fluxo sanguíneo foi significativamente menor durante a RCP com o LUCAS (16\%) do que durante a RCP manual (35\%). Com isso, concluiu-se que as compressões torácicas mecânicas fornecidas pelo dispositivo LUCAS melhoram a qualidade da RCP, reduzindo significativamente a fração sem fluxo sanguíneo e melhorando a qualidade da compressão torácica em comparação com a RCP manual.

Em seu estudo, Couper K, et al. (2016) avaliou oito artigos, contendo nove estudos (689 participantes), nos quais as metanálises mostraram uma associação entre o uso de dispositivo de compressão torácica mecânica e melhora hospitalar ou sobrevida em 30 dias e sobrevida em curto prazo. Também houve evidências de melhorias nos resultados fisiológicos. Feito isso, observou-se que os dispositivos de compressão torácica mecânica podem melhorar a evolução do paciente, quando usados em parada cardíaca hospitalar. No entanto, a qualidade das evidências atuais é muito baixa. Há uma necessidade de ensaios clínicos randomizados para avaliar o efeito na sobrevida em parada cardíaca hospitalar.

Desta forma, Kupershmidt S, et al. (2020), referiu que apesar dos benefícios hemodinâmicos associados ao dispositivo LUCAS-2 no laboratório e em outros ambientes, este e outros estudos indicam que a RCP por compressão manual supera a RCP assistida por automação durante a parada cardíaca extra-hospitalar. No entanto, os dados também sugerem que o treinamento aprimorado dos provedores de emergência para melhorar os tempos de resposta e os níveis de experiência com o equipamento pode melhorar os resultados associados ao LUCAS-2.

\section{CONSIDERAÇÕES FINAIS}

Apesar de um bom desempenho durante a compressão torácica, os dispositivos mecânicos não se mostraram mais efetivos que a técnica manual, pois não dependem apenas de fatores técnicos, mas também de fatores relacionados ao procedimento e a organização da equipe. Porém, quando utilizados por indivíduos capacitados em situações onde as compressões manuais consistentes não são praticáveis ou são perigosas para o socorrista, são uma boa opção. Além disso, quando esses dispositivos são utilizados, deve-se monitorizar de perto, pois sugerem um risco maior de algumas lesões, como pneumotórax e hematoma. Devese também ter uma atenção especial para reduzir o tempo sem compressões e atrasos para realização de outros procedimentos importantes na RCP, como a desfibrilação. 


\section{REFERÊNCIAS}

1. ANANTHARAMAN V, et al. Prompt use of mechanical cardiopulmonary resuscitation in out-of-hospital cardiac arrest: the MECCA study report. Singapore Med J, 2017; 58(7): 424-431.

2. BONNES JL, et al. Manual Cardiopulmonary Resuscitation Versus CPR Including a Mechanical Chest Compression Device in Out-of-Hospital Cardiac Arrest: A Comprehensive Meta-analysis From Randomized and Observational Studies. Ann Emerg Med, 2016; 67(3): 349-360.

3. COUPER K, et al. Mechanical devices for chest compression: to use or not to use. Curr Opin Crit Care, 2015; 21(3): 188-194.

4. COUPER K, et al. Mechanical chest compression devices at in-hospital cardiac arrest: A systematic review and metaanalysis. Resuscitation, 2016; 103: 24-31.

5. ESTOCK JL, et al. Comparison of chest compression interruption times across 2 automated devices: a randomized, crossover simulation study. Am J Emerg Med, 2016; 34(1): 57-62.

6. GATES S, et al. Mechanical chest compression for out of hospital cardiac arrest: Systematic review and metaanalysis. Resuscitation, 2015; 94: 91-97.

7. GATES S, et al. Prehospital randomised assessment of a mechanical compression device in out-of-hospital cardiac arrest (PARAMEDIC): a pragmatic, cluster randomised trial and economic evaluation. Health Technol Assess, 2017; 21(11): 1-176.

8. KHAN SU, et al. Efficacy and safety of mechanical versus manual compression in cardiac arrest - A Bayesian network meta-analysis. Resuscitation, 2018; 130: 182-188.

9. KOSTER RW, et al. Safety of mechanical chest compression devices AutoPulse and LUCAS in cardiac arrest: a randomized clinical trial for non-inferiority. Eur Heart J, 2017; 38(40): 3006-3013.

10. KUPERSHMIDT S, et al. Survival from Out-of-Hospital Cardiac Arrest - Comparing the Automated LUCAS-2 Device and Manual CPR. S D Med, 2020; 73(4): 171-177.

11. LIU M, et al. Mechanical chest compression with LUCAS device does not improve clinical outcome in out-of-hospital CARDIAC arrest patients: A systematic review and meta-analysis. Medicine (Baltimore), 2019; 98(44): e17550.

12. MARTI J, et al. The cost-effectiveness of a mechanical compression device in out-of-hospital cardiac arrest. Resuscitation, 2017; 117: 1-7.

13. MILLING L, et al. Prehospital cardiopulmonary resuscitation with manual or mechanical chest compression: A study of compression-induced injuries. Acta Anaesthesiol Scand, 2019; 63(6): 789-795.

14. POOLE K, et al. Mechanical CPR: Who? When? How?. Crit Care, 2018; 22(1): 140.

15. RUSSO A, et al. Utilizzo del massaggiatore automatico esterno nell'arresto cardiaco refrattario sul territorio e in ospedale [The use of mechanical chest compression devices for both out-of-hospital and in-hospital refractory cardiac arrest]. G Ital Cardiol (Rome), 2017; 18(4): 305-312.

16. TRANBERG T, et al. Quality of cardiopulmonary resuscitation in out-of-hospital cardiac arrest before and after introduction of a mechanical chest compression device, LUCAS-2; a prospective, observational study. Scand $\mathrm{J}$ Trauma Resusc Emerg Med, 2015; 23: 37.

17. WANG PL, Brooks SC. Mechanical versus manual chest compressions for cardiac arrest. Cochrane Database Syst Rev, 2018; 8(8):

18. WINTHER K, BLEEG RC. LUCAS ( $\left.{ }^{\mathrm{TM}}\right) 2$ in Danish Search and Rescue Helicopters. Air Med J, 2016; 35(2): 79-83.

19. WROE PC, et al. Emergency department use of a mechanical chest compression device frequently causes unanticipated interruptions in cardiopulmonary resuscitation. Resuscitation, 2018; 133: 3-4.

20. ZHU N, et al. A meta-analysis of the resuscitative effects of mechanical and manual chest compression in out-ofhospital cardiac arrest patients. Crit Care, 2019; 23(1): 100. 